\title{
LINEARIZATION FOR THE BOLTZMANN EQUATION
}

\author{
BY \\ F. ALBERTO GRUNBAUM( $\left.{ }^{1}\right)$
}

\begin{abstract}
In this paper we compare the nonlinear Boltzmann equation appearing in the kinetic theory of gases, with its linearized version. We exhibit an intertwining operator for the two semigroups involved. We do not assume from the reader any familiarity with Boltzmann's equation but rather start from scratch.
\end{abstract}

0. Introduction. Consider a dilute gas composed of a very large number of molecules moving in space according to the laws of classical mechanics, and colliding in pairs from time to time. Assume that we can disregard all external effects, such as gravity, so that the motion is completely specified by giving the intermolecular forces.

One is interested in the number of molecules which at time $t$ have position $r$ and velocity $v$, within $d r d v$. This is given by

$$
n(t, r, v)=N f(t, r, v) d r d v\left({ }^{2}\right)
$$

where $f$ is called the density function. It is clear that this quantity is going to change in time due to the motion of the molecules and to the effect of the collisions.

Boltzmann derived an equation for the rate of change of $f$ with time. It has the form of a nonlinear integro-differential equation:

$$
\begin{aligned}
\frac{\partial f}{\partial t}+v_{1} \frac{\partial f}{\partial r} & =B f \\
& =\iint\left[f\left(v_{2}^{*}\right) f\left(v_{1}^{*}\right)-f\left(v_{1}\right) f\left(v_{2}\right)\right]\left|v_{1}-v_{2}\right| I\left(\left|v_{1}-v_{2}\right|, \theta\right) \sin \theta d \theta d \phi d v_{2} .
\end{aligned}
$$

Here $f$ stands for $f\left(t, r, v_{1}\right)$. The integral part, containing the nonlinearity, reflects

Received by the editors February 19, 1971.

AMS 1970 subject classifications. Primary 45M05, 47H15, 82A40; Secondary 33A65, $60 \mathrm{~K} 35$.

Key words and phrases. Boltzmann equation, spatially homogeneous case, nonlinear equation, linearized version, intertwining operator, eigenvalue inequalities.

(1) The author wishes to express his deep gratitude to his thesis advisor Professor Henry P. McKean, Jr., under whose guidance this work was carried out. He has encouraged and helped the author all along this research and has made many contributions to make this a more readable work. These results are contained in the author's doctoral dissertation at The Rockefeller University, and were rewritten while the author was supported by the AEC under Contract AT(30-1)-1480-V.

${ }^{(2)} N$ is the total number of molecules. 
the effect of the collisions between molecules; the term $v_{1} \partial f / \partial r$ reflects the motion of the molecules between collisions. The integrals on the right will now be explained.

The intermolecular forces are supposed to be given by pair forces, all interactions involving more than two particles being ignored. Therefore, we deal first with the mechanism of an individual collision of two molecules. For the forthcoming description, it is convenient to refer the two colliding molecules, travelling with velocities $v_{1}$ and $v_{2}$, to the center-of-mass coordinate system. Thus the situation becomes that of a fictitious molecule, travelling with velocity $v=v_{2}-v_{1}$, scattered by a center of force placed at the center-of-mass, denoted by $O$; see the figure.

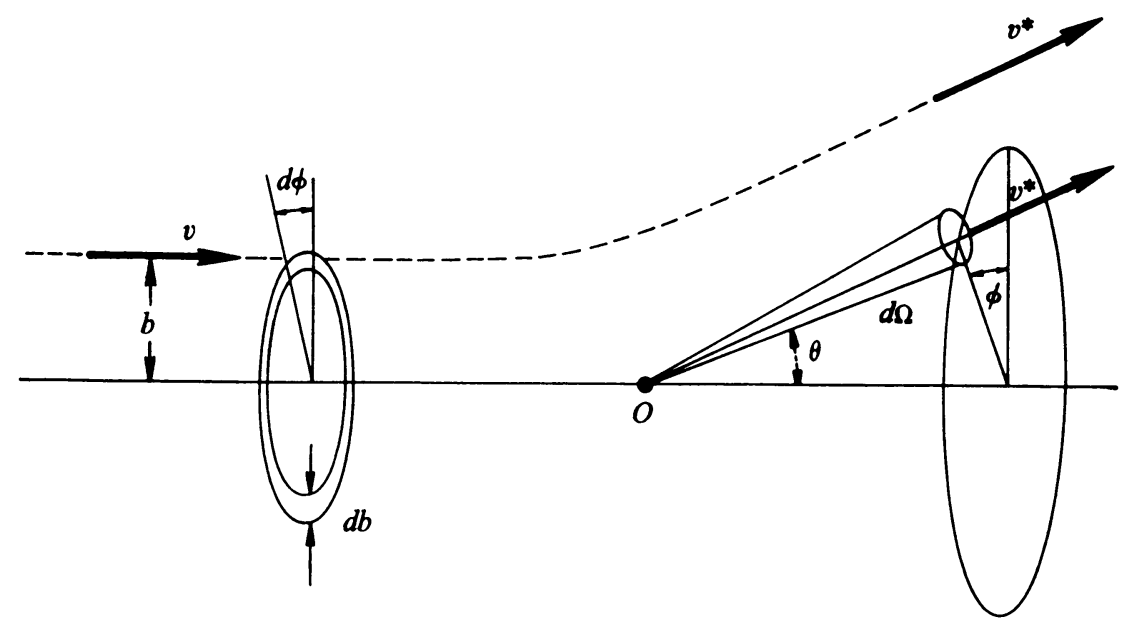

Recall that if $v_{1}^{*}, v_{2}^{*}$ denote the velocities of the molecules after the collision, we have the conservation laws of

momentum: $v_{1}+v_{2}=v_{1}^{*}+v_{2}^{*}$,

and energy: $\left|v_{1}\right|^{2}+\left|v_{2}\right|^{2}=\left|v_{1}^{*}\right|^{2}+\left|v_{2}^{*}\right|^{2}$,

and their consequence: $\left|v_{1}-v_{2}\right|=\left|v_{1}^{*}-v_{2}^{*}\right|$.

If one also recalls that $v=v_{2}-v_{1}$ and $v^{*}=v_{2}^{*}-v_{1}^{*}$ lie in the same plane, then it is clear that the impact parameter $b$ and the angles $\phi$ and $\theta$ in the figure determine the kinematics of a collision completely. The impact parameter $b$ measures the distance from the direction given by $v, 0 \leqq \phi<2 \pi$ is the azimuth angle, and $0 \leqq \theta \leqq \pi$ is the colatitude which measures the scattering angle formed by $v$ and $v^{*}$, see the figure.

Think now of a uniform beam of molecules coming from the left with velocity $v$. Then the dynamical description of the collision process, pictured here as a scattering process, is embodied in the expression

$$
\left|v_{1}-v_{2}\right| b d b d \phi=\left|v_{1}-v_{2}\right| I\left(\left|v_{1}-v_{2}\right|, \theta\right) \sin \theta d \theta d \phi
$$

which gives the number of molecules that pass through the shaded area in the 
figure, per unit time. This formula defines the differential scattering cross section $I$. Because $\theta$ can be computed from $b, v$, and the intermolecular force, $I$ is uniquely determined by this recipe.

As to the meaning of the integral itself, the number of collisions, in unit time, of a molecule with velocity $v_{1}$ with a molecule of (arbitrary) velocity $v_{2}$ and parameters $b$ and $\theta$, within $d b d \phi$, is given by

$$
\left|v_{1}-v_{2}\right| f\left(v_{1}, t\right) b d b d \phi .
$$

Boltzmann goes one step further and puts for the loss, due to collisions, of molecules with velocities $v_{1}$, within $d v_{1}$, in unit time,

$$
d v_{1} \iiint\left|v_{1}-v_{2}\right| f\left(v_{1}, t\right) f\left(v_{2}, t\right) b d b d \phi d v_{2} \text {. }
$$

Now he argues that a direct collision from $v_{1}, v_{2}$ into $v_{1}^{*}, v_{2}^{*}$ is allied to a restituting collision from $v_{1}^{*}, v_{2}^{*}$ into $v_{1}, v_{2}$ and that both stand on the same footing. Thus the gain of molecules with velocity $v_{1}$, in unit time, should be

$$
d v_{1}^{*} \iiint\left|v_{1}^{*}-v_{2}^{*}\right| f\left(v_{1}^{*}, t\right) f\left(v_{2}^{*}, t\right) b d b d \phi d v_{2}^{*} .
$$

To get the expression for $B f$ given by (1), it is now enough to combine (3) and (4) after appropriate use is made of the identity $d v_{1} d v_{2}=d v_{1}^{*} d v_{2}^{*}$ and the fact that $\left|v_{1}-v_{2}\right| I\left(\left|v_{1}-v_{2}\right|, \theta\right) \sin \theta d \theta d \phi$ remains unchanged if $v_{1} v_{2}$ are interchanged with $v_{1}^{*} v_{2}^{*}$. This says that $a$ transition from $\left(v_{1}, v_{2}\right)$ into $\left(v_{1}^{*}, v_{2}^{*}\right)$ has the same chance as one from $\left(v_{1}^{*}, v_{2}^{*}\right)$ into $\left(v_{1}, v_{2}\right)$, a fact which is used over and over again in this paper.

We emphasize that we have not presented a derivation from first principles. We computed the Stoss-zahl-ansatz (2) by invoking a dynamical description whose connection with actual molecules in a gas is not completely transparent. Then we accepted (3) on the basis of molecular chaos, and finally from (3) we arrived at (4) on the assumption of microscopic reversibility. The reader will find detailed explanations of these terms in Uhlenbeck-Ford [12] or Grad [3].

To conclude this look at $B f$, we remark that the total scattering cross section $\int I \sin \theta d \theta d \phi=\int b d b d \phi$ is convergent only if two molecules at a distance larger than some $R<\infty$ cannot feel each other. In that case, we have $\int b d b d \phi=\pi R^{2}$. This kind of restriction is usually referred to as a cutoff interaction.

Although the form of the equation (1) does not depend on the intermolecular force, the quantity $I\left(\left|v_{1}-v_{2}\right|, \theta\right)$ inside the integral does so. For the moment we mention only the cases of hard spheres and the Maxwellian gas. In the first case, the molecules are hard spheres which do not interact with each other except when they touch. Then they exchange their velocities in a perfectly elastic collision and one gets $I\left(\left|v_{1}-v_{2}\right|, \theta\right)=$ a constant multiple of $\sin (\theta / 2)$. In the Maxwellian case there is a central potential which is inversely proportional to $r^{4}$, and you find that $\left|v_{1}-v_{2}\right| I\left(\left|v_{1}-v_{2}\right|, \theta\right)$ is a function of $\theta$ alone with a pole at $\theta=0$. The pole is customarily removed by making a cutoff. 
There are many features of equation (1) -and ( $\left(1^{\prime}\right)$ below-which do not depend on the intermolecular force, for example:

(a) The Maxwell-Boltzmann densities

$$
g(v)=\left(2 \pi \sigma^{2}\right)^{-3 / 2} \exp \left(-|v|^{2} / 2 \sigma^{2}\right)
$$

are the only stationary densities for $\left(1^{\prime}\right)$.

(b) The first and second moments of the density function $f(t, v)$ are preserved in time, i.e. $\int|v| f(t, v) d v$ and $\int|v|^{2} f(t, v) d v$ are constant.

(c) The $H$-theorem, which states that the entropy $H$,

$$
H(t)=-\int f(t, v) \log f(t, v) d v,
$$

increases with time.

For all these facts, as well as for related material, the reader can consult Uhlenbeck-Ford [12] or Huang [7].

We will be concerned with a special instance of (1): the so-called spatially homogeneous case in which $f(t, r, v)$ is independent of $r$, and (1) simplifies to

$$
\partial f / \partial t=B f
$$

We also assume the interaction to have a cutoff, so that $\int I \sin \theta d \theta d \phi=\pi R^{2}<\infty$.

Under these restrictions the initial value problem for the Boltzmann equation has been much studied. The ideal result here would be that a finite second moment for the initial distribution assures existence and uniqueness of the solution.

The problem turns out to be quite a difficult one. Carleman [1] solved the case of radial solutions for the hard spheres case and Wild [13] the case of a cutoff Maxwellian gas $\left({ }^{3}\right)$. Povzner studied a slightly modified equation, which reduces in the spatially homogeneous case to the classical equation with a finite total scattering cross section (cutoff). He proves that a finite second moment for the initial distribution does guarantee existence, but he has to impose a finite fourth moment to get uniqueness. See [11].

The aim of this paper is to study the approach to equilibrium for $\left(1^{\prime}\right)$ in a very detailed way.

Instead of considering the general Boltzmann equation, we deal only with a one-dimensional caricature of the Maxwellian gas introduced and studied by Kac [8]. This model has all the mathematical features that are present in the actual Maxwellian gas but all manipulations are simpler. From a physical point of view, it has the disadvantage that in a collision, only energy, but not momentum, is preserved.

For this simple example you can give a very complete geometrical description of the convergence to the Maxwell-Boltzmann distribution. All considerations are now localized near this Maxwell-Boltzmann distribution, denoted here by $g$.

( $\left.{ }^{3}\right)$ For the space dependent case, the only one of physical interest, consult Grad [4]. 
First we prove existence and uniqueness of the solution for the nonlinear equation in some appropriate $L^{2}$ space. Then we see that the rate of approach io $g$ is governed by the first negative eigenvalue of the linearization of $B$ at $g$, i.e. $\left\|f_{t}-g\right\|$ $\leqq$ constant $\times e^{-v t}$. We also prove that we have a contractive flow, i.e. $\left\|f_{t}^{(1)}-f_{t}^{(2)}\right\|$ is a decreasing function of $t$ if $f^{(1)}$ and $f^{(2)}$ are close enough to $g$.

But much more than this can be said. We prove that there is an extremely smooth (actually analytic) nonlinear "change of coordinates" around $g$, so that if the nonlinear problem is reexpressed in terms of these new coordinates, then it is exactly the linearized problem. In this picture the result mentioned above about the rate of convergence appears as an innocent manifestation of the fact just mentioned.

Some straightforward extensions of this to include higher-order collisions are indicated in Appendix II. There one also finds a discussion of the difficulties in trying to extend the results to the general Boltzmann equation $\left(1^{\prime}\right)$.

The problem of the rate of approach to equilibrium for the solutions of the (nonlinear) Boltzmann equation has been treated by Grad [4] and McKean [9]. Grad shows that for a general spatially homogeneous case, the decay in some appropriate $L^{2}$ space is exponential and that the decay exponent can be taken as close as you please to the first negative eigenvalue of the linearized operator, provided the initial distribution is close enough to equilibrium. For Kac's caricature of the Maxwellian gas, McKean gets an exponential decay for the $L^{1}$ norm; but his decay constant is much smaller than it should be. On the other hand the $L^{1}$ norm, and not the one used by Grad or by us, is the one that makes more sense globally.

1. Kac's model of the Boltzmann equation. The purpose of this section is twofold. First, we introduce Kac's equation and give a condensed exposition of the $L^{1}(R)$ theory going with it. This space is the natural place to look at Kac's equation because the solution is a density function and $\int f(=1)<\infty$ is automatic.

Once this is done, we try to prepare the spirit of the reader to take up in $\$ \S 2-5$ the study of the "change of coordinates" described in the introduction.

For all details omitted in this section and for a very interesting analysis of many questions not touched by us, e.g. the central limit theorem for Maxwellian molecules, the reader may consult McKean [9].

We consider the initial value problem for a probability density function $f(t, a)$ :

$$
\frac{\partial f}{\partial t}(t, a)=\int_{R} \int_{S^{\prime}}\left[f\left(t, a^{*}\right) f\left(t, b^{*}\right)-f(t, a) f(t, b)\right] d b d w=B f
$$

subject to the conditions

$$
f(0, a)=f_{0} \geqq 0, \quad \int f(t, a) d a \equiv 1, \quad \sigma^{2}(f)=\int a^{2} f(t, a) d a<\infty .
$$

$\left.{ }^{4}\right) S^{\prime}$ is the circle $0 \leqq 0<2 \pi, R$ is the line. 
Here $d w=I(\theta) d \theta$ is a probability measure on $S^{\prime}$ with (Lebesgue) density $I(\theta)$, and

$$
a^{*}=a \cos \theta-b \sin \theta, \quad b^{*}=a \sin \theta+b \cos \theta .
$$

Usually the time dependence of $f$ will not be written out explicitly.

McKean adapted a construction first used by Wild [13] for the 3-dimensional Maxwellian gas to express the solution of problem (1) as a weighted sum of "products" of the initial datum $f_{0}$ with itself.

For that construction, one rewrites the Boltzmann equation in the form

$$
\partial f / \partial t=f * f-f
$$

where we define

$$
\left(f_{1} * f_{2}\right)(a)=\iint f_{1}\left(a^{*}\right) f_{2}\left(b^{*}\right) d b d w
$$

for functions belonging to the class $D$ given by

$$
D: \quad 0 \leqq f, \quad \int f=1, \quad \sigma^{2}(f)=\int f(a) a^{2} d a<\infty .
$$

This product maps $D \times D$ into itself, and for a general $I(\theta)$ is neither commutative nor associative. The first property can be achieved only if one imposes some symmetry on $I(\theta)$. To see this, one rewrites $f_{1} * f_{2}$ as

$$
\left(f_{1} * f_{2}\right)(a)=\iint_{a^{* 2}+b^{* 2}>a^{2}} \frac{f_{1}\left(a^{*}\right) f_{2}\left(b^{*}\right)}{\left(a^{* 2}+b^{* 2}-a^{2}\right)^{1 / 2}} I\left(\theta\left(a^{*}, b^{*}\right)\right) d a^{*} d b^{*}
$$

so that is clear that commutativity is equivalent to $I\left(\theta\left(a^{*}, b^{*}\right)\right)$ being a symmetric function of the pair $\left(a^{*}, b^{*}\right)$. One can see that this amounts to the condition $I(\pi / 4-\alpha)=I(\pi / 4+\alpha), 0 \leqq \alpha<2 \pi$. The nonassociativity of the product cannot be remedied by assumptions on $I(\theta)$ and it is an essential ingredient of the problem $\left({ }^{6}\right)$.

Without going into the details, see McKean [9], we mention that if the product defined in (3) were associative, one could express the solution of (2) as a Wild's sum

$$
f=\sum_{n=1}^{\infty} e^{-t}\left(1-e^{-t}\right)^{n-1} f_{0} * \cdots * f_{0} \quad(n \text {-fold }) .
$$

In our case, the associativity does not hold and the $n$-fold products in (4) must be interpreted by putting parentheses between the factors in all possible ways and averaging. Each term of Wild's sum is positive and it is easy to see that it converges in $L^{1}(R)$ for all $t \geqq 0$. One can also see that it gives a solution of (1a). No other solution of (1) exists since Wild's sum can be seen to be the smallest one, and its integral is already 1 . One can also check that $\sigma^{2}(f)=\sigma^{2}\left(f_{0}\right)$.

$\left(^{5}\right)$ For a fixed $a, \theta=\theta\left(a^{*}, b^{*}\right)$ is uniquely determined as the proper rotation needed to go from $\left(a^{*}, b^{*}\right)$ in $R^{2}$ to $(a, b)$ for some $b$.

$\left({ }^{\theta}\right)$ For instance, if $I(\theta) \equiv 1 / 2 \pi$, the product given by (3) is commutative but not associative. 
This completes our review of the $L^{1}(R)$ situation for (1) and we now turn to the study of the approach to equilibrium.

If the entropy of a probability density function is defined as $H[f]=-\int f \log f$, the $H$-theorem says that this quantity will increase in time, if $f$ is governed by (1) Gibbs' lemma [9] states that the maximum value of the entropy, computed on the class of all probability density functions with a given second moment, is assumed only by the Gaussian with mean zero $\left({ }^{7}\right)$. These two facts have been taken as a proof of the approach of the solutions of (1) to an equilibrium (Gaussian) distribution. See, for instance, Uhlenbeck-Ford [12]. For technical details needed to get a complete proof consult McKean [9] for Kac's model, Carleman [1] for a 3dimensional gas of hard balls, and Grad [4] for a wide class of cutoff potentials.

A detailed study of this approach to equilibrium for Kac's model is the subject of Chapter I. Our main concern will be to study the nonlinear evolution given by (1) or (2) "close" to the equilibrium position $g$ and to compare it with the much simpler evolution that one gets by linearizing the equation (1) around this equilibrium position. More explicitly, if we put $f=g+h$ and note that $g * g=g$, we can express (2) as

$$
\dot{f}=f * f-f=(g * h+h * g-h)+h * h
$$

or equivalently

$$
h=A h+h * h \text {. }
$$

$A$ is here the linear operator mapping $h$ into $g * h+h * g-h$, and $h * h$ is a quadratic correction term. The linearized evolution referred to above is given by ignoring $h * h$ in equation (5), thus obtaining

$$
h_{t}=e^{t A} h(0)
$$

or

$$
f_{t}=g+h_{t}
$$

This evolution (6) is to be compared, close to $f=g$, with the one given by the actual solution of (2).

Although we have seen that the initial value problem (2) is well posed in $L^{1}(R)$, we will find it convenient to work in a subspace of $L^{1}(R)$, namely, $L^{2}\left(g^{-1}\right)\left({ }^{8}\right)$. The fact that this is a Hilbert space will facilitate the comparison between the nonlinear and the linearized flows.

In the next section, we will prove that the problem (1) is also well posed in $L^{2}\left(g^{-1}\right)$ and we complete this section with some comments on our choice of this space.

(7) For example, $g(a)=(2 \pi)^{-1 / 2} \exp \left(-a^{2} / 2\right)$ if the second moment is put equal to one. We use $g$ from now on to denote this particular Gaussian density function.

$\left.{ }^{8}\right) L^{2}\left(g^{-1}\right)=L^{2}\left((2 \pi)^{1 / 2} \exp \left(a^{2} / 2\right)\right)$ is the space of all measurable functions $f(a)$ such that $\|f\|^{2}=(2 \pi)^{1 / 2} \int f^{2}(a) \exp \left(a^{2} / 2\right) d a<\infty$. It is clearly a subspace of $L^{1}(R)$. 
The distance from an arbitrary function in $L^{2}\left(g^{-1}\right)$ to the Gaussian $g(a)$ $=(2 \pi)^{-1 / 2} \exp \left(-a^{2} / 2\right)$ is given by

$$
\|f-g\|^{2}=\int(f-g)^{2}(a) g^{-1}(a) d a .
$$

This distance does not make much sense from the point of view of statistical mechanics, see [12]. But we may see that for $f$ close to the Gaussian $g$ it is related in a rather natural way to the entropy of $f$. Namely, if $f$ satisfies $\int f d a=\int g d a$ and $\sigma^{2}(f)=\sigma^{2}(g)$ and we write $f=g+h$, we have

$$
\begin{aligned}
H[f] & =-\int f \log f=-\int(g+h) \log g\left(1+\frac{h}{g}\right) \\
& =-\int g \log g-\int h \log g-\int(g+h)\left(-\frac{h}{g}+\frac{1}{2}\left(\frac{h}{g}\right)^{2}-\cdots\right) \\
& =H[g]+c_{1} \int h+c_{2} \int h(a) a^{2} d a+\int h^{2} g^{-1}+\text { higher order terms in } h \\
& =H[g]+\frac{1}{2} \int h^{2} g^{-1}+\text { "smaller" terms. }
\end{aligned}
$$

Here we used the fact that both $\int h d a$ and $\sigma^{2}(h)$ vanish. Therefore, we can say that $\|f-g\|^{2}=\int h^{2} g^{-1}$ gives the first-order correction to the expansion of the entropy of $f$ about the point $g$.

2. Existence and uniqueness for the even flow. We consider in this section Kac's model of the Boltzmann equation posed in $L^{2}\left(g^{-1}\right)$. In the previous section we noticed that there was a special subset of $L^{1}(R)$ where the problem was well posed, to wit: positive functions with integral 1 and a fixed variance ( 1 for instance).

In the same spirit, what we will see now is that Kac's equation is well posed in the subset of $L^{2}\left(g^{-1}\right)$ given by those functions with integral 1 and variance 1 . It will follow that if we also ask that $f$ be positive, we will still have a well-posed problem.

We state now the theorem to be proved in this section. It constitutes the backbone of all that comes after $\S 2$. For simplicity we assume that $I(\theta)=I(-\theta)$, a natural restriction on physical grounds. In fact, it says that the chance of a collision that takes velocities $(a, b)$ into $\left(a^{*}, b^{*}\right)$ equals the chance of one with the reversed effect. This feature of the Boltzmann equation was referred to in the introduction as "microscopic reversibility."

THEOREM 1. On the submanifold of $L^{2}\left(g^{-1}\right)$ given by $\int f(a) d a=1, \sigma^{2}(f)=1$, the initial value problem $\dot{f}=f * f-f$ is well posed in a sufficiently small ball $C$ centered about $g$. This means that there exists only one family of operators $Q_{t}, t \geqq 0$, mapping $C$ into itself and satisfying, for each $f_{0} \in C$,

(1) $Q_{t} f_{0} \in L^{2}\left(g^{-1}\right), \int\left(Q_{t} f_{0}\right)(a) d a \equiv 1, \sigma^{2}\left(Q_{t} f_{0}\right) \equiv 1$,

(2) $Q_{0} f_{0}=f_{0}, Q_{t_{1}+t_{2}} f_{0}=Q_{t_{2}} Q_{t_{1}} f_{0}$ and

$$
\text { strong } \lim _{t \rightarrow 0} t^{-1}\left[Q_{t} f_{0}-f_{0}\right]=f_{0} * f_{0}-f_{0}=B f_{0} \text {. }
$$


Moreover we have

(3) $\left\|Q_{t} f_{0}-g\right\| \leqq$ constant $\times e^{-v t}$, in which $-v$ is the top (negative) eigenvalue of the linearization of $B f$ at $g$,

(4) $\left\|Q_{t} f_{0}^{(1)}-Q_{t} f_{0}^{(2)}\right\| \leqq\left\|f_{0}^{(1)}-f_{0}^{(2)}\right\|$ if $f_{0}^{(1)}$ and $f_{0}^{(2)}$ belong to $C$.

The actual proof comes at the end of this section.

To discuss this problem, we make recourse to a formal series expansion of $f$ in terms of the orthogonal basis for the space $L^{2}\left(g^{-1}\right)$ given by the Hermite functions

$$
h_{n}(a)=\exp \left(-a^{2} / 2\right) H_{n}(a)=(-1)^{n} D^{n} \exp \left(-a^{2} / 2\right), \quad n \geqq 0 .
$$

Recall that

$$
\begin{array}{rlrl}
\int_{-\infty}^{\infty} h_{i}(a) h_{j}(a) g^{-1}(a) d a & =0 & & \text { for } i \neq j, \\
& =i ! 2 \pi & \text { for } i=j .
\end{array}
$$

We express our equation

$$
\dot{f}=f * f-f
$$

in terms of the basis $h_{n}(n \geqq 0)$ in a purely formal way at first. Expand $f=f_{t}$ as

$$
f=\sum f_{n}(t) h_{n}
$$

and assume that it is legitimate to write

$$
\partial f / \partial t=\sum \dot{f}_{n}(t) h_{n}
$$

To get the componentwise version of (7), we use the fundamental relation $\left.{ }^{9}\right)$, see Kac [6],

$$
\left(h_{\imath} * h_{j}\right)=(2 \pi)^{1 / 2} \int \cos ^{i} \theta \sin ^{j} \theta I(\theta) d \theta h_{i+j}
$$

satisfied by the Hermite functions in connection with the product defined in $(1.3)\left({ }^{10}\right)$.

From (9) and (10) we get the desired formula

$$
f_{n}=(2 \pi)^{1 / 2} \sum_{i=0}^{n} f_{i} f_{n-1} \int \cos ^{i} \theta \sin ^{n-i} \theta I(\theta) d \theta-f_{n} .
$$

Observe that because of the assumption on $I(\theta)$ mentioned at the beginning of this section, all integrals of the type

$$
\int \cos ^{\text {odd }} \theta \sin ^{\text {odd }} \theta I(\theta) d \theta \text { and } \int \cos ^{\text {even }} \theta \sin ^{\text {odd }} \theta I(\theta) d \theta
$$

( ${ }^{9}$ A proof of this relation and of a generalization of it are found in Appendix I.

${ }^{(10)}$ This means equation (3) in $\$ 1$, and the same convention is used throughout. 
will vanish, and (11) gets simplified into an even system:

$$
f_{2 n}=(2 \pi)^{1 / 2} \sum_{i=0}^{n} f_{2 i} f_{2 n-2 i} \int \cos ^{2 i} \theta \sin ^{2 n-2 i} \theta I(\theta) d \theta-f_{2 n},
$$

and an odd system:

$$
f_{2 n+1}=(2 \pi)^{1 / 2} \sum_{i=0}^{n} f_{2 i+1} f_{2 n-2 i} \int \cos ^{2 i+1} \theta \sin ^{2 n-2 i} \theta I(\theta) d \theta-f_{2 n+1} .
$$

Note that a little more symmetry (esp., $I(\theta) \equiv 1 / 2 \pi$ ) makes all the integrals in the odd system (11b) vanish too. We can therefore summarize the situation for (11a), (11b) as follows:

(a) the even components of $f$ evolve by themselves, according to a nonlinear system of the form $f_{\text {even }}=F\left(f_{\text {even }}\right)$;

(b) if we solve that system and insert the resulting even components into the odd system, the odd components evolve linearly according to an equation of form $f_{\text {odd }}=C(t) f_{\text {odd }}$.

One can prove that all the $f_{2 n+1}$ go to zero with $t$ going to $+\infty$; in particular, if we have the extra symmetry mentioned after (11b), we have simply

$$
f_{2 n+1}(t)=e^{-t} f_{2 n+1}(0) \text {. }
$$

This is the case when $I(\theta) \equiv 1 / 2 \pi$ for instance.

The interested reader can treat this linear system in the standard, but lengthy, way. A good reference for this is Hahn [5]. For the purpose at hand one can simply assume that $I(\theta)$ is a constant.

The main objective of this paper is a comparison between the nonlinear flow given by (7) or (11), and its linearization at $f=g=(2 \pi)^{-1 / 2} \exp \left(-a^{2} / 2\right)$. Therefore, we confine our attention for the rest of this chapter to the even flow given by (11a) and compare it with its linearization at $g$.

The restrictions, $\int f=1$ and $\sigma^{2}(f)=1$, mentioned at the beginning of this section, are expressed solely in terms of the even components of $f(a)$, namely

$$
\begin{aligned}
f_{0} & =\frac{1}{2 \pi} \int f(a) \exp \left(-a^{2} / 2\right)\left((2 \pi)^{1 / 2} \exp \left(a^{2} / 2\right)\right) d a=(2 \pi)^{-1 / 2} \quad\left({ }^{11}\right) \\
f_{2} & =\frac{1}{2 ! 2 \pi} \int f(a) \exp \left(-a^{2} / 2\right)\left(a^{2}-1\right)\left((2 \pi)^{1 / 2} \exp \left(a^{2} / 2\right)\right) d a \\
& =\frac{1}{4 \pi}\left((2 \pi)^{1 / 2}-(2 \pi)^{1 / 2}\right)=0 .
\end{aligned}
$$

Because of this we have to concentrate in the even flow given by (11a) on the submanifold described by

$$
f_{0}(t) \equiv(2 \pi)^{-1 / 2}, \quad f_{2}(t) \equiv 0,
$$

(11) One should not confuse this with the initial value of $f$. This is the only place where confusion may arise. 
and we have to prove that this problem is well posed close enough to $g(a)$ $=(2 \pi)^{-1 / 2} \exp \left(-a^{2} / 2\right)$.

We first make the linearization explicit by rewriting (11a), subject to (13), as

$$
\begin{aligned}
f_{2 n}= & \left(\int\left(\cos ^{2 n} \theta+\sin ^{2 n} \theta\right) I(\theta) d \theta-1\right) f_{2 n} \\
& +(2 \pi)^{1 / 2} \sum_{i=2}^{n-2} f_{2 i} f_{2 n-2 i} \int \cos ^{2 i} \theta \sin ^{2 n-2 i} \theta I(\theta) d \theta, \quad n \geqq 2 .
\end{aligned}
$$

Except for the fact that (14) makes only reference to the even components of $f$ with $n \geqq 2$, it is clear that it is identical to equation (1.5). If we use $x$ to denote the vector whose components are the even components of $f-g$ for $n \geqq 4$, that is

$$
x_{0}=x_{2}=0, \quad x_{2 n+1} \equiv 0, \quad x_{2 n}=(f-g)_{2 n} \quad(n \geqq 2),
$$

we can reexpress (14) in the succinct form

$$
\dot{x}=A x+x * x \text {. }
$$

Here $A$ is the diagonal operator acting on the space spanned by $h_{2 n}(n \geqq 2)$, by means of the rule

$$
A h_{2 n}=\left(\int\left(\cos ^{2 n} \theta+\sin ^{2 n} \theta\right) I(\theta) d \theta-1\right) h_{2 n}=\lambda_{2 n} h_{2 n}, \quad n \geqq 2 .
$$

It is plain that $A$ is selfadjoint with a totally discrete spectrum, ranging from $\lambda_{4}=\int\left(\cos ^{4} \theta+\sin ^{4} \theta\right) I(\theta) d \theta-1$ down to -1 , which is an accumulation point of the spectrum. For the case $I(\theta) \equiv 1 / 2 \pi$ that top negative eigenvalue is $-\frac{1}{4}$. To deal with (11a) subject to (13) rewritten as (14), we need

LEMMA 1. The nonlinear part in equation (14) satisfies a Lipschitz condition:

$$
\|x * x-y * y\| \leqq 2\|x-y\| \max (\|x\|,\|y\|) \text {. }
$$

Proof. If we could prove

$$
\|x * y\| \leqq\|x\|\|y\|,
$$

then we could get (16) by means of the following string of inequalities:

$$
\begin{aligned}
\|x * x-y * y\| & \leqq\|x *(x-y)\|+\|(x-y) * y\| \\
& \leqq\|x-y\|(\|x\|+\|y\|) \leqq 2\|x-y\| \max (\|x\|,\|y\|) .
\end{aligned}
$$

The square of the norm of an even vector $x=\sum_{i \geqq 2} x_{2 i} h_{2 i}$ in $L^{2}\left(g^{-1}\right)$ is given by $\sum_{i \geqq 2} x_{2 i}^{2}(2 i) ! 2 \pi$ and therefore we have from (13)

$$
\begin{aligned}
\|x * y\|^{2}= & \sum_{n=2}^{\infty}(2 \pi)(2 n) !\left[(2 \pi)^{1 / 2} \sum_{i=2}^{n-2} x_{2 i} y_{2 n-2 i} \int \cos ^{2 i} \theta \sin ^{2 n-2 i} \theta I(\theta) d \theta\right]^{2} \\
\leqq & (2 \pi)^{2} \sum_{n=2}^{\infty}\left(\sum_{i=2}^{n-2}(2 i) ! x_{2 i}^{2}(2 n-2 i) ! y_{2 n-2 i}^{2}\right) \\
& \cdot \sum_{j=2}^{n-2}\left(\begin{array}{c}
2 n \\
2 j
\end{array}\right)\left[\int \cos ^{2 j} \theta \sin ^{2 n-2 j} \theta I(\theta) d \theta\right]^{2}
\end{aligned}
$$

where we made use of the Schwarz inequality. 
Now we notice that

$$
\begin{aligned}
& \sum_{j=2}^{n-2}\left(\begin{array}{l}
2 n \\
2 j
\end{array}\right)\left[\int \cos ^{2 j} \theta \sin ^{2 n-2 j} \theta I(\theta) d \theta\right]^{2} \\
& =\sum_{j=2}^{n-2}\left(\begin{array}{l}
2 n \\
2 j
\end{array}\right) \iint\left(\cos ^{2 j} \alpha \sin ^{2 n-2 j} \alpha \cos ^{2 j} \beta \sin ^{2 n-2 j} \beta\right) I(\alpha) I(\beta) d \alpha d \beta \\
& =\iint(\cos \alpha \cos \beta+\sin \alpha \sin \beta)^{2 n} I(\alpha) I(\beta) d a d \beta \\
& =\iint \cos ^{2 n}(\alpha-\beta) I(\alpha) I(\beta) d \alpha d \beta \leqq 1
\end{aligned}
$$

Observe that we made use of the property $I(\theta)=I(-\theta)$ in the third line, to pull the sum inside of the integral. If we apply this to our previous inequality (18), we get

$$
\|x * y\| \leqq(2 \pi)^{2} \sum_{n=2}^{\infty} \sum_{i=2}^{n-2}(2 i) ! x_{2 i}^{2}(2 n-2 i) ! y_{2 n-2 i}^{2}=\|x\|^{2}\|y\|^{2} .
$$

This completes the proof of (16).

We are now in a position to prove that (14) is well posed. Recall that for $I(\theta)$ $\equiv 1 / 2 \pi$ the top eigenvalue of $A$ is $-\frac{1}{4}$. We consider this case only, but it should be plain that the proof has to be trivially modified to deal with a different $I(\theta)$.

LEMMA 2. There exists a unique solution to equation.(14) if $\|x(0)\|$ is small enough. Moreover $\|x(t)\| \leqq C e^{-t / 4}$.

Proof. Equation (14) is replaced by the integral equation

$$
x(t)=e^{t A} x(0)+\int_{0}^{t} e^{(t-s) A}(x(s) * x(s)) d s \equiv H(x(t)) .
$$

We are interested in a fixed point of this map because any solution of (19) is such a point, so we must first find a domain mapped into itself by $H$.

Take $E$ to be the set of those continuous functions $x(t)$ from $[0, \infty)$ into the subspace of $L^{2}\left(g^{-1}\right)$ spanned by $h_{2 n}(n \geqq 2)$, such that $\|x(t)\| \leqq \gamma \exp (-t / 4)$ for all positive $t$ and $\|x(0)\| \leqq \gamma^{2}$, with a constant $\gamma$ to be specified later on.

Recalling that the spectrum of $A$ lies to the left of $-\frac{1}{4}$ and using the proof of the previous lemma, we get

$$
\begin{aligned}
\|H x(t)\| & \leqq e^{-t / 4}\|x(0)\|+\int_{0}^{t} e^{-(t-s) / 4}\|x(s)\|^{2} d s \\
& \leqq e^{-t / 4}\|x(0)\|+\gamma^{2} \int_{0}^{t} e^{-(t-s) / 4} e^{-s / 2} d s \\
& \leqq e^{-t / 4}\|x(0)\|+4 \gamma^{2} e^{-t / 4}=e^{-t / 4}\left(\|x(0)\|+4 \gamma^{2}\right),
\end{aligned}
$$

so that $\|H x(t)\| \leqq 5 \gamma^{2} \exp (-t / 4)$, and this is smaller than $\gamma \exp (-t / 4)$ if $\gamma \leqq 1 / 10$. For any such $\gamma$, the corresponding class $E$ is sent into itself by the map $H$. Note that $H x(0)=x(0)$. 
We now construct a series which formally solves the fixed-point problem $H x=x$. The task is then to prove its convergence.

Take $x^{(1)}(t)=e^{t A} x(0), x^{(i)}=H x^{(i-1)}$ and define

$$
x=\sum_{i=1}^{\infty}\left[x^{(i+1)}-x^{(i)}\right]+x^{(1)} .
$$

Observe that, for $\gamma \leqq 1 / 10$, each $x^{(i)}$ lies in $E$ whenever $\|x(0)\| \leqq \gamma^{2}$. Define

$$
\begin{aligned}
& y_{i}(t)=\max \left(\left\|x^{(i)}(t)\right\|,\left\|x^{(i-1)}(t)\right\|\right), \\
& z_{i}(t)=\max _{t \leqq s}\left\|x^{(i)}(s)-x^{(i-1)}(s)\right\| .
\end{aligned}
$$

We know that

$$
y_{i}(s) \leqq \gamma e^{-s / 4}, \quad z_{1}(t)=\max _{t \leqq s}\left\|x^{(1)}(t)\right\| \leqq e^{-t / 4}\|x(0)\|,
$$

and

$$
\begin{aligned}
z_{i+1}(t) & =\max _{t \leqq s}\left\|H x^{i}(s)-x^{i}(s)\right\| \\
= & \max _{t \leqq s} \| e^{s A}\left[x^{(i-1)}(0)-x^{(i-2)}(0)\right] \\
& \quad+\int_{0}^{s} e^{(s-\alpha) A}\left(x^{(i-1)}(\alpha) * x^{(i-1)}(\alpha)-x^{(i-2)}(\alpha) * x^{(i-2)}(\alpha)\right) d \alpha \| \\
= & \left.\max _{t \leqq s} \| \int_{0}^{s} e^{(s-\alpha) A}\left(x^{(i-1)}(\alpha) * x^{(i-1)}\right)(\alpha)-x^{(i-2)}(\alpha) * x^{(i-2)}(\alpha)\right) d \alpha \| \\
& \leqq 2 \max _{t \leqq s} \int_{0}^{s} e^{-(s-\alpha) / 4} z_{i}(\alpha) y_{i}(\alpha) d \alpha .
\end{aligned}
$$

In the last line we used Lemma 1 of this section.

Now we make the inductive hypothesis that there exists a number $q$ such that

$$
z_{i}(s) \leqq\|x(0)\| q^{i-1} e^{-s / 4} .
$$

This is true for $i=1$, and by induction we get

$$
\begin{aligned}
z_{i+1}(t) & \leqq 2\|x(0)\| q^{i-1} \gamma \max _{t \leqq s} \int_{0}^{s} e^{-(s-\alpha) / 4} e^{-\alpha / 2} d \alpha \\
& \leqq 8\|x(0)\| q^{i-1} \gamma e^{-t / 4},
\end{aligned}
$$

so that if we start with $q=8 \gamma$ (which we know is smaller than one), we would have $z_{i+1}(t) \leqq\|x(0)\| q^{i} e^{-t / 4}$ proving the inductive hypothesis and making our series (20) absolutely convergent:

$$
\|x\| \leqq \sum\left\|x^{(i+1)}-x^{(i)}\right\|+\left\|x^{(1)}\right\| \leqq\|x(0)\| e^{-t / 4} \sum_{i=0}^{\infty} q^{i}<\infty .
$$

In the same vein, one goes on to prove that the series gives a solution of the original differential equation (14) and that this solution is unique. If the reader needs help at this point he can consult Hahn [5]. This ends the proof of Lemma 2. 
Putting together Lemmas 1 and 2 with the expression (12), we have a complete proof of the main theorem of this section for the case $I(\theta) \equiv \frac{1}{2} \pi$. Lemma 1 is the essential ingredient for part (4) of the theorem.

To prove parts (1) and (2) of that theorem for a nonconstant $I(\theta)$, one has to deal also with the linear odd system (11b).

3. The linearization problem. Suppose we have an equation of the type

$$
\dot{x}=A x+F(x)
$$

where $A$ is linear and $F$ analytic, with neither constant nor linear terms. Very close to $x=0$, the two vector fields $A x$ and $A x+F(x)$ look very much the same. A natural question is how is this reflected in the flows corresponding to (1a) and

$$
\dot{x}=A x .
$$

When $x$ lies in $R^{n}$ or $C^{n}$ this question was answered first by Poincaré [10]. He proved that if $\lambda_{1}, \lambda_{2}, \ldots, \lambda_{n}$ are the eigenvalues of $A$ and if

(a) $\lambda_{i} \neq \sum m_{j} \lambda_{j}$ for any positive integers $m_{j}$ with $\sum m_{j}>1$, and

(b) all the $\lambda_{j}$ lie on one side of a line passing through the origin,

then there exists an analytic map $\psi$ which is locally invertible and linearizes the flow near the origin. This means that if $Q_{t}$ denotes the map sending the initial data $x$ into the solution $x(t)$ at time $t$, for (1a) and if $T_{t}$ denotes the corresponding map for (1b), then $\psi$ intertwines $Q_{t}$ and $T_{t}$ in the sense that

$$
Q_{t}=\psi^{-1} \circ T_{t} \circ \psi
$$

near the origin.

If we want such a change of coordinates to exist for $A$ irrespective of the $F$, then conditions (a) and (b) are probably necessary, as an example by Hartman [6] seems to indicate. Much less, nearly nothing in fact, is required if we are contented with a smooth, but not analytic, $\psi$. See Hartman [6].

In $\$ 5$ we will present such a change of coordinates $\psi$ for the even part of Kac's equation, see (2.11a). Of course the space is now infinite dimensional. In general, this is bound to produce difficulties. They can be overcome in our case because $A$ has a (negative) pure point spectrum which accumulates at the point $-\int I(\theta) d \theta$ $=-1$. See the remarks after $\left(2.14^{\prime}\right)$.

It comes as an unexpected bonus that $\psi$ can be expressed as

$$
\psi=\underset{t \rightarrow+\infty}{\operatorname{strong}} \lim T_{-t} Q_{t}
$$

A nice and trivial consequence of this fact is that for each small enough $x$, there exists a vector $\psi(x)$ so that $T_{-t}\left(T_{t} \psi(x)-Q_{t} x\right)$ goes to zero as $t \rightarrow \infty$. Clearly $\psi$ is characterized by this property. A brief discussion of the relations between (2) and (3) in the case of $R^{2}$ will clarify the situation. 
For simplicity, let us consider only the case of a symmetric operator $A$. It is simple to prove that if the limit (3) exists and has an inverse, then the $\psi$ so defined intertwines $T_{t}$ and $Q_{t}$ according to (2). On the other hand, simple examples show that not all intertwining maps $\psi$ can be computed by means of (3). Now we will see where the trouble lies and indicate a way to modify (3) to get an intertwining map in general.

First we express Poincaré's condition (a) in terms of $T_{t}$. Take $A$ to be $A=\left(\begin{array}{ll}\alpha & 0 \\ 0 & \beta\end{array}\right)$ and consider the formal power series

$$
W(x, y)=\left(\sum a_{i j} x^{i} y^{j}, \sum b_{i j} x^{i} y^{j}\right)=(x, y)+\text { higher order terms. }
$$

Then we have

$$
T_{-t} W T_{t}(x, y)=\left(\sum a_{i j} x^{i} y^{j} e^{(i \alpha+j \beta-\alpha) t}, \sum b_{i} x^{i} y^{j} e^{(i \alpha+j \beta-\beta) t}\right)
$$

and it is now plain that condition (a) is precisely what is needed for an arbitrary $W$ of the above form to be able to split $T_{-t} W T_{\imath}$ into $I$ plus a couple of formal power series $T_{-t} W T_{t}=I+W_{+}(t)+W_{-}(t)$ where

(4a) each term of $W_{+}(t)$ converges to 0 as $t \rightarrow+\infty$,

(4b) each term of $W_{-}(t)$ converges to 0 as $t \rightarrow-\infty$.

To understand why (2) will not imply (3) in general, suppose that $A$ satisfies Poincaré's conditions so that there exists an analytic map $\psi$ satisfying (2). Because of the previous comments, we can write

$$
T_{-t} Q_{t}=T_{-t} \psi^{-1} T_{t} \psi=\left(I+\psi_{+}^{-1}(t)+\psi_{-}^{-1}(t)\right) \psi=\psi+\psi_{+}^{-1}(t) \psi+\psi_{-}^{-1}(t) \psi .
$$

Clearly (3) will exist only when $\psi_{-}^{-1}(t) \psi \equiv 0$.

Expression (5) also suggests how to get an intertwining map for $\psi$ for the pair $T_{t}, Q_{t}$. One has to split the formal power series expansion of $T_{-t} Q_{t}$ into three pieces

$$
T_{-t} Q_{t}=\psi+\psi_{1}(t)+\psi_{2}(t)
$$

where $\psi_{1}(t)$ and $\psi_{2}(t)$ satisfy (4a) and (4b) respectively. To check formally that the $\psi$ so constructed does the trick, (2) is straightforward. Thus the real problem is to check convergence of this formal series for $\psi$.

4. Eigenvalue inequalities. In this section we prove an elementary inequality which surprisingly contains the core of the future development. From it we derive important inequalities concerning the eigenvalues of the linear transformation $A$ defined in (2.15).

LEMMA. If $x_{1}^{2}+x_{2}^{2}=1$, then for every $n, m \geqq 2$, we have

$$
\left(-1+\sum_{i=1}^{2} x_{i}^{2 n+2 m}\right)>\left(-1+\sum_{i=1}^{2} x_{i}^{2 n}\right)+\left(-1+\sum_{i=1}^{2} x_{i}^{2 m}\right)
$$

$\left.{ }^{(12}\right)$ We disregard the trivial case when one of the $x_{i}=0$. The proof is not the simplest one, and is made so as to extend naturally to the case when there are more than two $x_{i}$ 's; see Appendix II. 
Proof. For a fixed $\left(x_{i}\right)$ satisfying the assumptions, consider the function $h(n)$ $=1-\sum_{i=1}^{2} x_{i}^{2 n}$. Our aim is to prove that $h(n)$ is subadditive. For that purpose we look at the derivative (with respect to $n$ ) of the function $n^{-1} h(n)$ :

$$
\left(n^{-1} h(n)\right)^{\cdot}=n^{-2}\left[\sum_{i=1}^{2} x_{i}^{2 n}-1-\sum_{i=1}^{2} x_{i}^{2 n} \log x_{i}^{2 n}\right] \equiv n^{-2} r(n) .
$$

One checks that $r(n)$ is a decreasing function of $n$, so that $n^{-1} h(n)$ will be increasing up to some value of $n$ and decreasing from there on.

Assume for a moment that we could prove

$$
h(2) / 2>h(4) / 4 \text {. }
$$

From here and the previous comments, it is clear that $n^{-1} h(n)$ would be decreasing at least from $n=3$ on. Therefore for $n, m \geqq 2$ we would have

$$
h(n+m)=\frac{n}{n+m} h(n+m)+\frac{m}{n+m} h(n+m)<n \frac{h(n)}{n}+m \frac{h(m)}{m}=h(n)+h(m)
$$

and the lemma would be proved.

Thus we have only to show that (2) holds. This is equivalent to showing that $2\left(1-x^{2}-(1-x)^{2}\right)>1-x^{4}-(1-x)^{4}$ for $0<x<1$ and this is trivially verified.

Now we can prove the

THEOREM 2. Irrespective of the angular scattering density $I(\theta)$, the eigenvalues of the linear operator $A$ defined in (2.15) satisfy

$$
\lambda_{2 n}+\lambda_{2 m}<\lambda_{2 n+2 m}
$$

Proof. Recall that

$$
\lambda_{2 n}=\int\left(\cos ^{2 n} \theta+\sin ^{2 n} \theta-1\right) I(\theta) d \theta,
$$

and then notice that except for $\theta=0, \pi / 2, \pi, \frac{3}{2} \pi$ we have strict inequality for the integrands involved in (3). This proves the theorem.

We can say a little more than (3) if we notice that when $n$ grows to $+\infty, \lambda_{2 n}$ tends to -1 ; namely, there exists a $c>0$ so that

$$
\lambda_{2 n+2 m}-\left(\lambda_{2 n}+\lambda_{2 m}\right) \geqq c>0
$$

independently of $n, m \geqq 2$.

5. Intertwining operator. Most of the work up to this point was of a preparatory nature. In $\S 2$, we singled out the even system (11a) describing the nonlinear piece of equation (2.11); it is of the type

$$
\dot{x}=A x+x * x .
$$

Here $x$ is a vector belonging to the subspace of $L^{2}\left(g^{-1}\right)$ spanned by $h_{2 n}: n \geqq 2, A$ is explicitly given in (2.15), and the $*$ product is defined in (1.3). 
The point $x=0$ is critical for (1), and we know from $\S 2$ that solutions exist and are unique nearby. Thus, we can speak of the semigroup $Q_{t}, t \geqq 0$, relating initial data $x=x(0)$ to the solution $x(t)$ at time $t$, at least if $\|x\|$ is small enough.

A much simpler evolution is obtained by ignoring the nonlinear part $x * x$ in (1):

$$
\dot{x}=A x \text {. }
$$

The solution of this problem is given by $T_{t}=e^{t A}$ acting upon $x=x(0)$. The exponential makes good sense, because $A$ is selfadjoint and negative definite.

The purpose of this section is to prove

THEOREM 3. If $\|x\|$ is small enough, the limit

$$
\psi(x)=\underset{t \rightarrow+\infty}{\operatorname{strong} \lim } T_{-t} Q_{t} x
$$

exists and belongs to the $L^{2}\left(g^{-1}\right)$ span of $h_{2 n}(n \geqq 2)$. Moreover, the map $x \rightarrow \psi(x)$ is analytic close to $x=0$ with an analytic inverse, and

$$
Q_{t}=\psi^{-1} T_{t} \psi
$$

We first introduce some auxiliary material and prove two lemmas.

Given operators $K$ and $L$ acting upon $x$, define $K * L$ to be the operator $K * L: x \rightarrow(K x) *(L x)$; then (1) and (2) become

$$
\begin{aligned}
& \dot{Q}_{t} x=A Q_{t} x+\left(Q_{t} * Q_{t}\right) x, \\
& \dot{T}_{t} x=A T_{t} x
\end{aligned}
$$

Now

$$
S_{t}=T_{-t} Q_{t}
$$

can be easily shown to satisfy the equation

$$
\dot{S}_{t}=e^{-t A}\left(e^{t A} S_{t} * e^{t A} S_{t}\right), \quad S_{0}=I .
$$

This is equivalent to the integral equation

$$
S_{t}=I+\int_{0}^{t} e^{-s A}\left(e^{s A} S_{s} * e^{s A} S_{s}\right) d s,
$$

which we use to express $S_{t}$ as a sum of contributions of different numbers of factors. Namely, we put

$$
S_{t}=\sum_{n=1}^{\infty} R_{t}^{(n)}
$$

where the first three terms are

$$
\begin{aligned}
R_{t}^{(1)}= & I, \quad R_{t}^{(2)}=\int_{0}^{t} e^{-s A}\left(e^{s A} * e^{s A}\right) d s \\
R_{t}^{(3)}= & \int_{0}^{t} e^{-s A}\left(e^{s A} * e^{s A} \int_{0}^{s} e^{-\xi A}\left(e^{\xi A} * e^{\xi A}\right) d \xi\right) d s \\
& +\int_{0}^{t} e^{-s A}\left(e^{s A} \int_{0}^{s} e^{-\xi A}\left(e^{\xi A} * e^{\xi A}\right) d \xi * e^{s A}\right) d s
\end{aligned}
$$


Now by induction, if we have defined $R_{t}^{(i)}$ for all $i<n$, we construct $R_{t}^{(n)}$ in the following way:

(a) split $n$ in all possible ways as $j+(n-j) ; j=1, \ldots, n-1$,

(b) define $R_{t}^{(n)}$ as the sum of all possible pieces of the form

$$
\int_{0}^{t} e^{-s A}\left(e^{s A} R_{s}^{(j)} * e^{s A} R_{s}^{(n-j)}\right) d s
$$

Having defined $R_{t}^{(n)}, n \geqq 1$, in this way we can now check formally that $S_{t}$ defined by (9) solves (8). In fact

$$
e^{-t A}\left(e^{t A} S_{t} * e^{t A} S_{t}\right)=\sum_{n=1}^{\infty}\left(\sum_{j=1}^{n-1}\left(e^{-t A} R^{j} * e^{t A} R^{n-j}\right)\right)=S_{t}
$$

and clearly $S_{0}=I$. To make use (and sense) of the expression $S_{t}=\sum_{n=1}^{\infty} R_{t}^{(n)}$, we need to prove that this formal series actually converges in $L^{2}\left(g^{-1}\right)$, and therefore gives a bona fide solution of (8).

For that end we prove

LEMMA 1. There exists a constant $c>0$ such that, for all positive $t$ and all $x, y$ in the $L^{2}\left(g^{-1}\right)$ span of $h_{2 n}(n \geqq 2)$,

$$
\left\|e^{-s A}\left(e^{s A} x * e^{s A} y\right)\right\| \leqq e^{-c s}\|x\|\|y\| .
$$

Proof. By (2.15)

$$
\begin{aligned}
e^{s A} h_{2 i} * e^{s A} h_{2 j} & =\exp \left(\left(\lambda_{2 i}+\lambda_{2 j}\right) s\right) h_{2 i} * h_{2 j} \\
& =(2 \pi)^{1 / 2} \int \cos ^{2 i} \theta \sin ^{2 j} \theta I(\theta) d \theta \exp \left(\left(\lambda_{2 i}+\lambda_{2 j}\right) s\right) h_{2 i+2 j}
\end{aligned}
$$

Thus

$$
\begin{aligned}
& e^{-s A}\left(e^{s A} h_{2 i} * e^{s A} h_{2 j}\right) \\
& \quad=2 \pi \int \cos ^{2 i} \theta \sin ^{2 j} \theta I(\theta) d \theta \exp \left(\left(\lambda_{2 i}+\lambda_{2 j}-\lambda_{2 i+2 j}\right) s\right) h_{2 i+2 j}
\end{aligned}
$$

If we now put $x=\sum_{i \geq 2} \alpha_{2 i} h_{2 i}, y=\sum_{i \geq_{2}} \beta_{2 i} h_{2 i}$, and use (12), we get

$$
\begin{aligned}
& e^{-s A}\left(e^{s A} x * e^{s A} y\right) \\
& =(2 \pi)^{1 / 2} \sum_{n=2}^{\infty} h_{2 n} \sum_{i=2}^{n-2} \alpha_{2 i} \beta_{2 n-2 i} \int \cos ^{2 i} \theta \sin ^{2 j} \theta I(\theta) d \theta \exp \left(\lambda_{2 i}+\lambda_{2 n-2 i}-\lambda_{2 n}\right) s .
\end{aligned}
$$

Bring in now the inequalities (4.4) to get $-\lambda_{2 n}+\left(\lambda_{2 i}+\lambda_{2 n-2 i}\right) \leqq-c$. Now this is used to get the bound

$$
\begin{aligned}
& \left\|e^{-s A}\left(e^{s A} x * e^{s A} y\right)\right\|^{2} \\
& \quad \leqq 2 \pi \sum_{n=2}^{\infty}(2 n) !\left[\sum_{i=2}^{n-2}(2 \pi)^{1 / 2} \alpha_{2 i} \beta_{2 n-2 i} \int \cos ^{2 i} \theta \sin ^{2 n-2 i} \theta I(\theta) d \theta\right]^{2} e^{-2 c s} \\
& \leqq e^{-2 c s}\|x\|^{2}\|y\|^{2} .
\end{aligned}
$$

In the last line we used the same way of estimating as in (2.18). The lemma is proved. 
The inequality just proved allows one to conclude uniqueness of the solution for $\left(8^{\prime}\right)$. Indeed if $S_{t}^{1}$ and $S_{t}^{2}$ were two solutions of $\left(8^{\prime}\right)$, one gets from (11) the relation:

$$
\left\|S_{t}^{1}-S_{t}^{2}\right\| \leqq \int_{0}^{t}\left(\left\|S_{s}^{1}\right\|+\left\|S_{s}^{2}\right\|\right) e^{-c s}\left\|S_{s}^{1}-S_{s}^{2}\right\| d s
$$

and from here one proceeds in a standard way.

A more direct approach consists in observing that equation $\left(8^{\prime}\right)$ is, except for the operator $e^{t A}$, equivalent to $\left(2.14^{\prime}\right)$.

We are now in a position to prove the crucial

\section{LEMMA 2.}

$$
\left\|R_{t}^{(n)} x\right\| \leqq\left(\left(1-e^{c t}\right) / c\right)^{n-1}\|x\|^{n} .
$$

Proof. We get the estimate (13) by induction on $n$. To begin with, $R_{t}^{(1)}=I$, so (13) is trivial for $n=1$. Now use (11) and the inductive hypothesis to get from the definition of $R_{t}^{(n)}$

$$
\begin{aligned}
\left\|R_{t}^{(n)} x\right\| & =\left\|\sum_{j=1}^{n-1} \int_{0}^{t} e^{s A}\left(e^{-s A} R_{s}^{(j)} x * e^{-s A} R_{s}^{(n-j)} x\right) d s\right\| \\
& \leqq \sum_{j=1}^{n-1} \int_{0}^{t}\|\| d s \leqq \sum_{j=1}^{n-1} \int_{0}^{t}\left(\frac{1-e^{-c s}}{c}\right)^{n-2} e^{-c s} d s\|x\|^{n} \\
& =\left(\frac{1-e^{-c t}}{c}\right)^{n-1}\|x\|^{n} .
\end{aligned}
$$

The lemma is proved.

Finally, we can use this lemma to estimate the formal sum (9) as follows: if $\|x\|<c$, we have for all $t \geqq 0$

$$
\left\|S_{t} x\right\| \leqq \sum_{1}^{\infty}\left\|R_{t}^{n} x\right\| \leqq \sum_{1}^{\infty}\left(\frac{1-e^{-c t}}{c}\right)^{n-1}\|x\|^{n}<\infty,
$$

and therefore $S_{t} x$ is well defined as the sum of an absolutely convergent series. Now we can complete the proof of Theorem 3 .

To get the existence of the limit (3), we estimate

$$
\left\|\sum_{n=1}^{\infty} R_{t_{2}}^{(n)} x-\sum_{n=1}^{\infty} R_{t_{1}}^{(n)} x\right\|
$$

as in (14). The result is that for $t_{1} \leqq t_{2} \leqq \infty,(15)$ is bounded by

$$
\begin{aligned}
\sum_{n=1}^{\infty}(n-1) \int_{t_{1}}^{t_{2}} e^{-c s}\left(\frac{1-e^{-c s}}{c}\right)^{n-2} d s\|x\|^{n}=\left.\sum_{n=1}^{\infty}\left(\frac{1-e^{-c t}}{c}\right)\right|_{t=t_{1}} ^{t=t_{2}}\|x\|^{n} \\
\leqq\left(\exp \left(-c t_{1}\right)-\exp \left(-c t_{2}\right)\right) \sum_{n=1}^{\infty}(n-1) \frac{\|x\|^{n}}{c^{n-1}}
\end{aligned}
$$


In the last step we used the fact that $\beta^{n}-\alpha^{n} \leqq n(\beta-\alpha)$ if $0 \leqq \alpha \leqq \beta \leqq 1, n \geqq 1$. Closely related estimates show that $\psi(x)$ is an analytic function of $x$, if $\|x\|<c$. This means that $\psi$ can be expressed as the sum of an absolutely convergent power series:

$$
\psi(x)=x^{(0)}+x^{(1)}+x^{(2)}+\cdots,
$$

where

$$
x_{n}^{(0)}=x_{n}, \quad x_{n}^{(1)}=\sum_{i=2}^{n-2} x_{2 i} x_{2 n-2 i} \int_{0}^{\infty} e^{-s A}\left(e^{s A} h_{2 i} * e^{s A} h_{2 n-2 i}\right) d s, \quad \ldots
$$

The radius of convergence of this series is then at least $c$, the constant appearing in the inequalities (4.4).

It is simple to use the estimates derived above to see that $\left\|\psi(x)-\sum_{k=0}^{n} x^{(k)}\right\| \rightarrow 0$ as $n \rightarrow \infty$, and $\sum_{k=0}^{\infty}\left\|x^{(k)}\right\|<\infty$ for $\|x\|<c$. Notice that

$$
T_{t} T_{-(t+s)} Q_{(t+s)}=T_{-s} Q_{i+s}=T_{-s} Q_{s} Q_{t}
$$

so that if we keep $t$ fixed, while $s \rightarrow \infty$, we get

$$
T_{t} \psi=\psi Q_{t}
$$

(4) would be immediate from (17) if $\psi(x)$ were invertible. The gradient of $\psi(x)$ at $x=0$ is the identity map, so the inverse function theorem for analytic functions, see Dieudonné [2], guarantees the existence and analyticity of $\psi^{-1}$ close to $x=0$. The theorem is proved.

We close this section by observing that the knowledge of $\psi$ comes close to determining the scattering density $I$. If we perform the indicated integrals in (16), we will get in the denominators of the quadratic part all possible combinations of the type $\lambda_{2 n}-\left(\lambda_{2 i}+\lambda_{2 n-2 i}\right)$.

This is actually enough to determine the spectrum $\lambda_{2 i}$ because we have $\lambda_{2 n} \rightarrow-1$ if $n \rightarrow \infty$. If we recall that

$$
\lambda_{2 i}=\int\left(\cos ^{2 i} \theta+\sin ^{2 i} \theta-1\right) I(\theta) d \theta,
$$

we see that to find $I$ we have to deal with a classical moment problem; especially, the quadratic part of $\psi$ suffices to determine

$$
I(\theta)+I(\pi-\theta), \quad 0 \leqq \theta<\pi .
$$

It is clear that the even flow cannot give us any information beyond (18). If we want to recover the whole of $I$, we have to deal also with the odd eigenvalues $\lambda_{2 n+1}$ :

$$
\lambda_{2 n+1}=\int\left(\cos ^{2 n+1} \theta+\sin ^{2 n+1} \theta-1\right) I(\theta) d \theta .
$$

The reader will easily see that the set of all eigenvalues determines the scattering density $I$ unequivocally. 
6. Remarks on more general cases. We want to put an end to this paper by indicating briefly how we could handle two different problems along the same lines.

The first problem might be of physical relevance. The second one is motivated by the hard-sphere model of the Boltzmann equation and poses serious mathematical difficulties not resolved here.

For the first problem, consider a gas undergoing multiple (instead of only binary) collisions. At the end of an exponentially distributed random time, $m$ particles collide together with probability $k_{m}$, with $\sum_{m \geqq 2}^{n} k_{m}=1$.

Consider the vector $\left(a_{1}, \ldots, a_{m}\right)$ comprising the velocities of the $m$ particles. For simplicity we consider only one-dimensional velocities. The effect of a collision of $m$ particles is a proper $m$-dimensional rotation

$$
0:\left(a_{1}, \ldots, a_{m}\right) \rightarrow\left(a_{1}^{*}, \ldots, a_{m}^{*}\right) \text {. }
$$

If we now distribute 0 according to the law $I_{m}(0) d 0$, we get a higher-order Boltzmann equation for the density $f\left(a_{1}\right)$ of the number of particles having velocity $a_{1}$ :

$$
\frac{\partial f}{\partial t}=\sum_{m=2}^{n} k_{m} f^{(m)}-f \text {. }
$$

Here

$$
f^{(m)}\left(a_{1}\right)=\int_{R^{m-1}} \int_{\mathrm{SO}(m)} f\left(a_{1}^{*}\right) f\left(a_{2}^{*}\right) \cdots f\left(a_{m}^{*}\right) I_{m}(0) d 0 d a_{2} \cdots d a_{m} .
$$

The reader should compare this with (1.1a).

If each of the densities $I_{m}$ has some symmetry properties (compare with the assumption $I(\theta)=I(-\theta)$ in $\S 2)$, we get the same splitting that we had for (2.11) into an even and odd system. For the case of $\$ 2$, we had the numbers

$$
\lambda_{2 i}=\int\left(\cos ^{2 i} \theta+\sin ^{2 i} \theta-1\right) I(\theta) d \theta
$$

as the eigenvalues for the generator of the linearized flow. The Hermite functions were the eigenfunctions. They are still eigenfunctions for the corresponding linear generator in this case, and the eigenvalues are changed to

$$
\lambda_{2 i}=\sum_{m=2}^{n} k_{m} \int_{x_{1}^{2}+\cdots+x_{m}^{2}=1}\left(x_{1}^{2 i}+\cdots+x_{m}^{2 i}-1\right) I_{m}(0) d 0
$$

where $x_{i}=O_{i i}, i=1, \ldots, m$. This assertion comes from the formula

(2) $\int \cdots \int h_{i_{1}}\left(a_{1}^{*}\right) \cdots h_{i_{m}}\left(a_{m}^{*}\right) I_{m}(0) d 0 d a_{2}, \ldots, d a_{m}=$ constant $\times h_{i_{1}+\cdots+i_{m}}\left(a_{1}\right)$

which follows from (5) in Appendix I. The restriction $x_{1}^{2}+\cdots+x_{m}^{2}=1$ implies, for all $i, j \geqq 2$,

(3) $\left(-1+x_{1}^{2 i+2 j}+\cdots+x_{m}^{2 i+2 j}\right) \geqq\left(-1+x_{1}^{2 i}+\cdots+x_{m}^{2 i}\right)+\left(-1+x_{1}^{2 j}+\cdots+x_{m}^{2 j}\right)$. 
For $m=2$, this is the lemma proved in $\S 4$, the general proof will be found in Appendix II. Using (3) one gets $\lambda_{2 i}+\lambda_{2 j}<\lambda_{2 i+2 j}$ for the $\lambda$ 's given by (1), and from here

$$
\lambda_{\sum 2 n_{i}}>\sum \lambda_{2 n_{i}}
$$

Using these inequalities (4), plus the fact that the spectrum of the generator of the linearized flow accumulates at $-\sum_{m=2}^{n} k_{m}=-1$, one can get a proof of the existence of $\psi=$ strong $\lim _{l \rightarrow \infty} T_{-t} Q_{t}$ in the same fashion as we did in $\S 5$.

This finishes our consideration of the first problem, and we look now at a different situation.

Consider a problem of the form

$$
\dot{x}=A x+x * x .
$$

Here $x$ belongs to some Hilbert space and $x * y$ is a bilinear product from $H \times H$ into $H$. Assume finally that existence and uniqueness can be proved for equation (5), so that it makes sense to speak of the semigroup of operators $Q_{t}$ satisfying $\dot{Q}_{t}=A Q_{t}+Q_{t} * Q_{t}$.

We take up again the problem of comparing $T_{t}=e^{t A}$ with $Q_{t}$. We are mainly interested in finding conditions on $A$ and the $*$ product guaranteeing the existence of $\psi=$ strong $\lim _{t \rightarrow \infty} T_{-i} Q_{t}$. In the previous sections we have seen how to deal with this problem in the case when $A$ has a purely discrete spectrum $\left(\lambda_{n}\right)$, with corresponding eigenfunctions $f_{n}$ : we have to express $f_{i} * f_{j}$ in terms of the set $f_{n}$, and if $f_{i} * f_{j}$ has a nonzero component in the $n$th direction, then we have to be sure that $\lambda_{n}-\left(\lambda_{i}+\lambda_{j}\right)>c>0$ for some $c$ independent of $i, j, n$.

In the same fashion, if $A$ has the spectral resolution $\int \lambda d E_{\lambda}$, and if we can find $a$ constant $c>0$ so that for every $\lambda, \mu$

$$
\left(I-E_{\lambda}\right) H *\left(I-E_{\mu}\right) H \subset\left(I-E_{\lambda+\mu+c}\right) H,
$$

then the limit $\psi=$ strong $\lim _{t \rightarrow \infty} T_{-t} Q_{t}$ exists.

We feel that one is perfectly justified in considering (6) as a rather useless condition. Much more ingenuity is clearly needed to find handier conditions to tackle the problem of the existence of the limit (5) in actual cases such as hard spheres.

Appendix I: Products of Hermite functions. Here we prove a property of the Hermite functions due to Kac [8]. Actually, we generalize the relation he found. Such a generalization was used in $\S 6$. Recall that the Hermite polynomials are defined as

$$
H_{n}(a)=(-1)^{n} \exp \left(a^{2} / 2\right) D^{n} \exp \left(-a^{2} / 2\right), \quad n=0,1,2, \ldots,
$$

and that this set is complete and orthogonal in $L^{2}(g)$ with

$$
\begin{aligned}
\int_{-\infty}^{\infty} \exp \left(-a^{2} / 2\right) H_{i}(a) H_{j}(a) d a & =0, & i \neq j, \\
& =i !(2 \pi)^{1 / 2}, & i=j .
\end{aligned}
$$


$\operatorname{LEMma}\left({ }^{13}\right)$. If $x_{1}^{2}+\cdots+x_{n}^{2}=1$, then

$$
\begin{aligned}
& H_{m}\left(x_{1} a_{1}+\cdots+x_{n} a_{n}\right) \\
& \quad=\sum_{m_{1}+\cdots+m_{n}=m}\left(\begin{array}{c}
m \\
m_{1} \cdots m_{n}
\end{array}\right) x_{1}^{m_{1}} \cdots x_{n}^{m_{n}} H_{m_{1}}\left(a_{1}\right) \cdots H_{m_{n}}\left(a_{n}\right) .
\end{aligned}
$$

Proof. It is convenient to think of the $n$-tuple $\left(a_{1}, \ldots, a_{n}\right)$ as a vector in $R^{n}$ denoted by $a$. Let $O$ be an orthogonal matrix having the numbers $x_{1}, \ldots, x_{n}$ as its first row. We define a map in $R^{n}$ by means of $a \rightarrow O a$. Setting $b=O a$, we observe that $\partial / \partial b_{1}=\sum x_{i} \partial / \partial a_{i}$ and that $\sum a_{i}^{2}=\sum b_{i}^{2}$. We know that

$$
\begin{aligned}
\left(x_{1} \partial / \partial a_{1}\right. & \left.+\cdots+x_{n} \partial / \partial a_{n}\right)^{m} \\
& =\sum_{m_{1}+\cdots+m_{n}=m}\left(\begin{array}{c}
m \\
m_{1} \cdots m_{n}
\end{array}\right) x_{1}^{m_{1}} \cdots x_{n}^{m_{n}} \frac{\partial^{m_{1}}}{\partial a_{1}^{m_{1}}} \cdots \frac{\partial^{m_{n}}}{\partial a_{n}^{m_{n}}} .
\end{aligned}
$$

Therefore, if we apply the right-hand operator in (4) to the function $\exp \left(-\frac{1}{2}|a|^{2}\right)$ and then multiply the result by $(-1)^{m} \exp \left(\frac{1}{2}|a|^{2}\right)$, we get the right-hand side of the equality (3).

But now the rest is clear, because the left-hand operator in (4) is $\partial^{m} / \partial b_{1}^{m}$. Thus, if we apply it to $\exp \left(-\frac{1}{2}|b|^{2}\right)=\exp \left(-\frac{1}{2}|a|^{2}\right)$ and then multiply the result by $(-1)^{m} \exp \left(\frac{1}{2}|b|^{2}\right)$, we get $H_{m}\left(b_{1}\right)$ on the left-hand side of (3). Finally $H_{m}\left(b_{1}\right)$ $=H_{m}\left(x_{1} a_{1}+\cdots+x_{n} a_{n}\right)$ and the lemma is proved.

Now we are in a position to prove

THEOREM 4. If $O=\left(x_{i j}\right)$ is an orthogonal matrix and if $a \in R^{n}$, then

$$
\begin{aligned}
& \int \cdots \int H_{i_{1}}\left((O a)_{1}\right) \cdots H_{i_{n}}\left((O a)_{n}\right) \exp \left(-\left(a_{1}^{2}+\cdots+a_{n}^{2}\right) / 2\right) d a_{2} \cdots d a_{n} \\
& =(2 \pi)^{(n-1) / 2} x_{11}^{i_{1}} \cdots x_{n 1}^{i_{n}} H_{i_{1}}+\cdots+i_{n}\left(a_{1}\right) \exp \left(-a_{1}^{2} / 2\right) .
\end{aligned}
$$

This result is due to Kac [8] for $n=2$.

Proof. Except for the factor $\exp \left(-a_{1}^{2} / 2\right)$ the integral in (5) is a polynomial in $a_{1}$ alone, and can therefore be written as

$$
\sum \alpha_{k} H_{k}\left(a_{1}\right) \exp \left(-a_{1}^{2} / 2\right) \text {. }
$$

We integrate this against $H_{m}\left(a_{1}\right) d a_{1}$ to pick out $\alpha_{m}$. In the left side of (5) we change $a$ into $O^{-1} a=O^{*} a$ so as to have

(6) $\int \cdots \int H_{i_{1}}\left(a_{1}\right) \cdots H_{i_{n}}\left(a_{n}\right) H_{m}\left(\left(O^{*} a\right)_{1}\right) \exp \left(-\left(a_{1}^{2}+\cdots+a_{n}^{2}\right) / 2\right) d a_{1} \cdots d a_{n}=\alpha_{m}$.

Now expand $H_{m}\left(\left(O^{*} a\right)_{1}\right)$ using the previous lemma, converting the left side of (6) into a sum of several integrals. Each one of these is an $n$-fold integral which splits as a product of one-variable integrals.

$\left({ }^{13}\right)$ This result is well known, but our proof is elementary. 
Using the orthogonality relations (2), we observe that most of these integrals vanish; the only one which gives a contribution is the one coming from the additive decomposition of $m$ as $m=i_{1}+\cdots+i_{n}$. This surviving integral can be easily performed, and the stated formula drops out.

For instance if $n=2$, which is Kac's case, we get

(7) $\int H_{i}\left(a^{*}\right) H,\left(b^{*}\right) \exp \left(-\left(a^{2}+b^{2}\right) / 2\right) d b=(2 \pi)^{1 / 2} \cos ^{i} \theta \sin ^{j} \theta H_{i+j}(a) \exp \left(-a^{2} / 2\right)$

in the notation of $\$ \S 1$ and 2.

If we integrate against $I(\theta) d \theta$ and use $h_{m}(a)=\exp \left(-a^{2} / 2\right) H_{m}(a)$ we find

$$
\left(h_{i} * h_{i}\right)(a)=(2 \pi)^{1 / 2} \int \cos ^{i} \theta \sin ^{j} \theta I(\theta) d \theta h_{\imath+j}(a)
$$

which coincides with (2.10). Formula (6.2) is derived in the same way.

Appendix II: An elementary inequality. Here we extend the lemma proved in $\S 4$.

LEMMA. If $x_{1}^{2}+\cdots+x_{k}^{2}=1$ and $n, m \geqq 2$, then

$$
\left(-1+\sum_{i=1}^{k} x_{i}^{2 n+2 m}\right) \geqq\left(-1+\sum_{i=1}^{k} x_{i}^{2 n}\right)+\left(-1+\sum_{i=1}^{k} x_{i}^{2 m}\right) \text {. }
$$

Proof. A look at the proof presented in $\$ 4$ will convince the reader that it is enough to check relation (4.2), i.e. $2 h(2)>h(4)$, where $h(n)$ is given in this case by $h(n)=1-\sum_{i=1}^{k} x_{i}^{2 n}$. We will actually prove that, at least for $k \geqq 3$,

$$
h(2) / 2>h(3) / 3
$$

which clearly suffices because of the comments of $\$ 4$.

Inequality (2) is equivalent to

$$
3 \sum_{i=1}^{k} x_{i}^{4}-2 \sum_{i=1}^{k} x_{i}^{6}<1
$$

under the restraint $\sum_{i=1}^{k} x_{i}^{2}=1$. Using Lagrange multipliers to maximize the left hand of $\left(2^{\prime}\right)$, we find that at such a maximum, the quantity $\omega=x_{i}^{2}\left(1-x_{i}^{2}\right), i=1, \ldots, k$, has to be independent of $i$. We want to conclude that all the $x_{i}$ 's have to coincide, and this is seen as follows. As a function of $x^{2}, x^{2}\left(1-x^{2}\right)$ takes the value $\omega$ once at a point $y^{2} \leqq \frac{1}{2}$ and once at the point $1-y^{2} \geqq \frac{1}{2}$. Because $k \geqq 3$ and $\sum_{i=1}^{k} x_{i}^{2}=1$, the $x_{i}$ 's have to coincide. Thus each one of the $x_{i}^{2}$ 's takes the value $1 / k$, and it is enough to check that $3(1 / k)^{2} k-2(1 / k)^{3} k<1$. Thus the lemma is proved.

\section{BIBLIOGRAPHY}

1. T. Carleman, Problèmes mathématiques dans la théorie cinétique des gaz, Publ. Sci. Inst. Mittag-Leffler, 2, Almqvist and Wiksells, Uppsala, 1957. MR 20 \#4935.

2. J. Dieudonné, Foundations of modern analysis, Pure and Appl. Math., vol. 10, Academic Press, New York, 1960. MR 22 \#11074. 
3. H. Grad, Principles of the kinetic theory of gases, Handbuch der Physik (herausgegeben von S. Flügge), Band 12, Thermodynamik der Gase, Springer-Verlag, Berlin, 1958, pp. 205-294. MR 24 \#B1583.

4. - Asymptotic equivalence of the Navier-Stokes and nonlinear Boltzmann equations, Proc. Sympos. Appl. Math., vol. 17, Amer. Math. Soc., Providence, R. I., 1965, pp. 154-183. MR 32 \#1979.

5. W. Hahn, Stability of motion, Die Grundlehren der math. Wissenschaften, Band 138, Springer-Verlag, New York, 1967. MR 36 \#6716.

6. P. Hartman, Ordinary differential equations, Wiley, New York, 1964. MR 30 \#1270.

7. K. Huang, Statistical mechanics, Wiley, New York, 1963. MR 27 \#4605.

8. M. Kac, Foundations of kinetic theory, Proc. Third Berkeley Sympos. on Math. Statist. and Prob., 1954/55, vol. 3, Univ. of California Press, Berkeley and Los Angeles, 1956, pp. 171-197. MR 18, 960.

9. H. P. McKean, Jr., Speed of approach to equilibrium for Kac's caricature of a Maxwellian gas, Arch. Rational Mech. Anal. 21 (1966), 343-367. MR 35 \#4963.

10. H. Poincaré, Sur les propiétés des fonctions définies par les équations aux différences partielles. Vol. 1, Gauthier-Villars, Paris, 1929.

11. A. Povzner, The Boltzmann equation in the kinetic theory of gases, Mat. Sb. 58 (100) (1962), 65-86; English transl., Amer. Math. Soc. Transl. (2) 47 (1965), 193-216. MR 25 \#5755.

12. G. Uhlenbeck and G. Ford, Lectures in statistical mechanics, Lectures in Appl. Math. (Proc. Summer Seminar, Boulder, Colorado, 1960), vol. 1, Amer. Math. Soc., Providence, R. I., 1963. MR 27 \#1241.

13. E. Wild, On Boltzmann's equation in the kinetic theory of gases, Proc. Cambridge Philos. Soc. 47 (1951), 602-609. MR 13, 195.

Courant Institute of Mathematical Sciences, New York University, New York, NEW YORK 10012 